\title{
PERIODIC POTENTIALS WITH MINIMAL ENERGY BANDS
}

\author{
MARK S. ASHBAUGH AND ROMAN SVIRSKY
}

(Communicated by Kenneth R. Meyer)

\begin{abstract}
We consider the problem of minimizing the width of the lowest band in the spectrum of Hill's equation, $-u^{\prime \prime}+q(x) u=\lambda u$ on $\mathbb{R}$ with $q(x+1)=q(x)$ for all $x \in \mathbb{R}$, when the potential function $q$ is allowed to vary over a ball of radius $M>0$ in $L^{\infty}$. We show that minimizing potentials $q_{*}$ exist and that, when considered as functions on the circle, they must have exactly one well on which $q_{*}(x)$ must equal $-M$ and one barrier on which $q_{*}(x)$ must equal $M$; these are the only values that $q_{*}$ can assume (up to changes on sets of measure zero). That is, on the circle there is a single interval where $q_{*}(x)=M$ and on the complementary interval $q_{*}(x)=-M$. These results can be used to solve the problem of minimizing the gap between the lowest Neumann eigenvalue and either the lowest Dirichlet eigenvalue or the second Neumann eigenvalue for the same equation restricted to the interval $[0,1]$.
\end{abstract}

In an earlier paper [4] the problem of minimizing or maximizing the gap between the two lowest Dirichlet eigenvalues of a general Schrödinger operator $-\Delta+V(x)$ on a bounded domain $\Omega \subset \mathbb{R}^{n}$ when the potential $V$ was subjected to a $p$-norm constraint $(1 \leq p \leq \infty)$ was considered. In this paper we show how the same ideas can be applied to minimize the band-width for the lowest energy band of Hill's equation and to minimize the gap between the two lowest Neumann (resp., first Neumann and first Dirichlet) eigenvalues in the case where the potential is subjected to an $L^{\infty}$ constraint. Work of a similar nature, but concerned only with a single eigenvalue, and upon which the developments in this paper and its precursor [4] are based, is to be found in [1-3].

We consider Hill's equation in the form

$$
-u^{\prime \prime}+q(x) u=\lambda u,
$$

where $q(x)$ is assumed to be measurable and periodic of period 1, that is, $q(x+1)=q(x)$ for all $x \in \mathbb{R}$. Following the notation of Magnus and Winkler [9] we have as characteristic values, $\left\{\lambda_{i}\right\}_{i=0}^{\infty}$ and $\left\{\lambda_{i}^{\prime}\right\}_{i=1}^{\infty}$, which obey

$$
\lambda_{0}<\lambda_{1}^{\prime} \leq \lambda_{2}^{\prime}<\lambda_{1} \leq \lambda_{2}<\lambda_{3}^{\prime} \leq \lambda_{4}^{\prime}<\lambda_{3} \leq \cdots .
$$

Here the $\lambda_{i}$ 's are the eigenvalues of equation $(1)$ on $[0,1]$ when periodic boundary conditions $\left(u(1)=u(0), u^{\prime}(1)=u^{\prime}(0)\right)$ are imposed and the $\lambda_{i}^{\prime}$ 's

Received by the editors April 16, 1990.

1980 Mathematics Subject Classification (1985 Revision). Primary 34B25, 34B30.

Key words and phrases. Hill's equation, eigenvalue gaps, band widths. 
are the eigenvalues of the same problem when antiperiodic boundary conditions $\left(u(1)=-u(0), u^{\prime}(1)=-u^{\prime}(0)\right)$ are imposed. Furthermore, we shall denote by $\left\{\mu_{i}\right\}_{i=1}^{\infty}$ and $\left\{\nu_{i}\right\}_{i=0}^{\infty}$ the Dirichlet and Neumann eigenvalues, respectively, for equation (1) on $[0,1]$ with Dirichlet (resp., Neumann) boundary conditions imposed at both endpoints. It is well known [5, 7] that the following inequalities hold:

$$
\begin{gathered}
\nu_{0} \leq \lambda_{0} ; \\
\lambda_{2 i-1}^{\prime} \leq \mu_{2 i-1}, \nu_{2 i-1} \leq \lambda_{2 i}^{\prime} \text { for } i=1,2,3, \ldots ; \\
\lambda_{2 i-1} \leq \mu_{2 i}, \nu_{2 i} \leq \lambda_{2 i} \text { for } i=1,2,3, \ldots
\end{gathered}
$$

In this terminology, the quantities with which we concern ourselves here are $\lambda_{1}^{\prime}-\lambda_{0}, \nu_{1}-\nu_{0}$, and $\mu_{1}-\nu_{0}$. As the results about $\nu_{1}-\nu_{0}$ and $\mu_{1}-\nu_{0}$ follow readily from those for $\lambda_{1}^{\prime}-\lambda_{0}$, we deal for some time mainly with $\lambda_{1}^{\prime}-\lambda_{0}$.

We begin by showing existence of a minimizing potential $q_{*}$. For $M>0$ we let $S(M)=\left\{q \in L^{\infty}(0,1) \mid\|q\|_{\infty} \leq M\right\}$ and argue first that $\lambda_{1}^{\prime}-\lambda_{0}$ (viewed as a function of the potential $q$ ) is bounded on this set. Indeed, this follows from the fact that $\lambda_{0}$ and $\lambda_{1}^{\prime}$ are both bounded there. Uniform upper bounds follow from the inequalities (2) and (3) above and the corresponding bounds for Dirichlet eigenvalues as found in $[4,6]$. For the lower bound one can use the estimate

$$
\begin{aligned}
\lambda_{0} & =\int_{0}^{1} u_{0}\left[-u_{0}^{\prime \prime}+q(x) u_{0}\right] d x \\
& =-\int_{0}^{1} u_{0} u_{0}^{\prime \prime} d x+\int_{0}^{1} q(x) u_{0}(x)^{2} d x \geq \lambda_{0}(q \equiv 0)-M=-M,
\end{aligned}
$$

where we have taken $u_{0}$ to be a normalized eigenfunction corresponding to $\lambda_{0}$ and where we used the Rayleigh-Ritz inequality to bound $-\int_{0}^{1} u_{0} u_{0}^{\prime \prime} d x$ below by $\lambda_{0}(q \equiv 0)$. Alternatively, we could obtain all necessary bounds simultaneously by using the operator inequalities

$$
-\frac{d^{2}}{d x^{2}}-M \leq-\frac{d^{2}}{d x^{2}}+q(x) \leq-\frac{d^{2}}{d x^{2}}+M,
$$

where each operator that appears here is taken to have periodic (resp., antiperiodic boundary conditions). From the min-max principle [10] it then follows that

$$
-M=\lambda_{0}(0)-M \leq \lambda_{0}(q) \leq \lambda_{0}(0)+M=M
$$

and

$$
\pi^{2}-M=\lambda_{1}^{\prime}(0)-M \leq \lambda_{1}^{\prime}(q) \leq \lambda_{1}^{\prime}(0)+M=\pi^{2}+M .
$$

We therefore have

Lemma 1. For $q \in S(M)$, there are bounds on each of $\lambda_{i}(q)$ and $\lambda_{i}^{\prime}(q)$ depending only on $M$. In particular, on $S(M), \lambda_{1}^{\prime}(q)-\lambda_{0}(q)$ is bounded uniformly in $q$ and explicitly we have

$$
\pi^{2}-2 M \leq \lambda_{1}^{\prime}(q)-\lambda_{0}(q) \leq \pi^{2}+2 M .
$$


The same bounds hold for the quantities $\mu_{1}(q)-\nu_{0}(q)$ and $\nu_{1}(q)-\nu_{0}(q)$ and, more generally, one has the analogous bounds

$$
\begin{aligned}
(4 m+1) \pi^{2}-2 M & \leq \lambda_{2 m+1}^{\prime}(q)-\lambda_{2 m}(q) \\
& \leq(4 m+1) \pi^{2}+2 M \text { for } m=0,1,2, \ldots
\end{aligned}
$$

and

$$
\begin{aligned}
(4 m-1) \pi^{2}-2 M & \leq \lambda_{2 m-1}(q)-\lambda_{2 m}^{\prime}(q) \\
& \leq(4 m-1) \pi^{2}+2 M \text { for } m=1,2,3, \ldots .
\end{aligned}
$$

The corresponding general results for Dirichlet and Neumann eigenvalues are

$$
\begin{aligned}
(2 m+1) \pi^{2}-2 M & \leq \nu_{m+1}(q)-\nu_{m}(q), \mu_{m+1}(q)-\nu_{m}(q) \\
& \leq(2 m+1) \pi^{2}+2 M \text { for } m=0,1,2, \ldots, \\
(2 m+1) \pi^{2}-2 M & \leq \mu_{m+1}(q)-\mu_{m}(q), \nu_{m+1}(q)-\mu_{m}(q) \\
& \leq(2 m+1) \pi^{2}+2 M \text { for } m=1,2,3, \ldots .
\end{aligned}
$$

Of course, in each of the inequalities (8)-(12) above one can replace the lower bound by 0 by virtue of the inequalities (3). The existence of a minimizing potential $q_{*}$ for the quantity $\lambda_{1}^{\prime}(q)-\lambda_{0}(q)$, (resp., for $\nu_{1}(q)-\nu_{0}(q)$ or $\mu_{1}(q)-$ $\left.\nu_{0}(q)\right), q \in S(M)$, now follows by fairly standard subsequencing arguments [3, 4]. Note, however, that no claim of uniqueness of the minimizing potential $q_{*}$ is made in any of the cases considered.

We now proceed to the main result of this paper, the characterization of the minimizing potentials $q_{*}$ for $\lambda_{1}^{\prime}-\lambda_{0}$.

Theorem 1. Let $M$ be a positive real number. Consider Hill's equation $-u^{\prime \prime}+$ $q(x) u=\lambda u$ on $\mathbb{R}$, where $q(x+1)=q(x)$ and $q \in \widetilde{S}(M) \equiv\{q \mid q(x+1)=q(x)$ and $\left.\|q\|_{\infty} \leq M\right\}$. Then there exists at least one potential $q_{*} \in \widetilde{S}(M)$ such that $\lambda_{1}^{\prime}(q)-\lambda_{0}(q)$ is minimized over $q \in \widetilde{S}(M)$ by $q=q_{*}$ and any such minimizing potential $q_{*}$ restricted to $[0,1]$ has the form

$$
q_{*}(x)= \pm M\left[\chi_{(a, b)}(x)-\chi_{[0,1] \backslash(a, b)}(x)\right],
$$

where $0 \leq a<b \leq 1$ (and $a=0, b=1$ cannot occur), to within equivalence of measurable functions. (Here $\chi_{A}(x)$ denotes the characteristic function of the set $A \subset \mathbb{R}$.) More specifically, if $u_{0}$ and $u_{1}$ denote normalized eigenfunctions for $\lambda_{0}$ and $\lambda_{1}^{\prime}$, respectively, then

$$
q_{*}(x)= \begin{cases}M & \text { a.e. for } x \in B_{+} \equiv\left\{x \in \mathbb{R} \mid u_{0}(x)^{2}>u_{1}(x)^{2}\right\}, \\ -M & \text { a.e. for } x \in B_{-} \equiv\left\{x \in \mathbb{R} \mid u_{0}(x)^{2}<u_{1}(x)^{2}\right\},\end{cases}
$$

and, furthermore, the sets $B_{ \pm}$are nonempty and $u_{1}$ is a nondegenerate eigenfunction. (In particular, $\lambda_{1}^{\prime}\left(q_{*}\right)$ is not degenerate with $\lambda_{2}^{\prime}\left(q_{*}\right)$ and therefore, by the inequalities (2), both are simple eigenvalues.)

Remark. There is never a unique minimizing potential (for any $M>0$ ) since all translates of a minimizer $q_{*}$ are also minimizers, and this implies nonuniqueness unless $q_{*} \equiv$ const. But $q_{*} \equiv$ const is impossible by our characterization above. A natural further question is whether or not $q_{*}$ is unique up to translation; however, this appears to be nontrivial to answer analytically and we do not consider it further here. On the other hand, it might be noted that our 
results here reduce this question to a point where it could easily be handled numerically.

Proof. Existence was discussed above so we need only deal with the question of characterizing $q_{*}$ here. Exactly as in [4] and as sketched in our Appendix below, it can be argued through the use of degenerate perturbation theory $[8,10]$ that $\lambda_{1}^{\prime}\left(q_{*}\right)$ must be nondegenerate. Since $\lambda_{0}$ is always nondegenerate, perturbation theory yields

$$
\left.\frac{d}{d \kappa}\left[\lambda_{1}^{\prime}\left(q_{*}+\kappa P\right)-\lambda_{0}\left(q_{*}+\kappa P\right)\right]\right|_{\kappa=0}=\int_{0}^{1} P(x)\left[u_{1}(x)^{2}-u_{0}(x)^{2}\right] d x,
$$

where we assume the eigenfunctions $u_{0}$ and $u_{1}$ to be normalized. Since by the assumed minimizing property of $q_{*}$ this derivative must not be negative for any perturbation $P$ such that $q_{*}+\kappa P \in \widetilde{S}(M)$ for some range $[0, \varepsilon), \varepsilon>0$, of $\kappa$, it follows that on $B_{+}=\left\{x \mid u_{0}(x)^{2}>u_{1}(x)^{2}\right\}$ all positive perturbations of $q_{*}$ must be inadmissible; hence, $q_{*}=M$ on $B_{+}$, and similarly on $B_{-}=$ $\left\{x \mid u_{0}(x)^{2}<u_{1}(x)^{2}\right\}, q_{*}=-M$. Since a straightforward argument (see [4]) shows that $u_{0}(x)^{2}=u_{1}(x)^{2}$ cannot hold on a set of positive measure, it follows that $q_{*}= \pm M$ except perhaps on a set of measure 0 . Thus

$$
\begin{aligned}
q_{*}(x) & =M\left[\chi_{B_{+}}(x)-\chi_{B_{-}}(x)\right] \\
& =M\left[\chi_{B_{+}}(x)-\chi_{B_{+}^{c}}(x)\right]=-M\left[\chi_{B_{-}}(x)-\chi_{B_{-}^{c}}(x)\right] \text { a.e. }
\end{aligned}
$$

(where the superscript " $c$ " denotes the complement in $\mathbb{R}$ ), which establishes equation (14). To establish equation (13) it remains only to show that one of $B_{+}$or $B_{-}$when restricted to the interval $[0,1]$ is just an interval $(a, b)$. We do this by showing that on any half-open interval of length $1, u_{0}(x)^{2}=u_{1}(x)^{2}$ exactly twice and hence, that $B_{+}$and $B_{-}$are both just single intervals when viewed as subsets of the circle $S^{1}$ (which we realize as $\mathbb{R} / \mathbb{Z}$ ).

To facilitate the discussion we translate the problem so that $u_{1}(0)=0=$ $u_{1}(1)$ (it is known that $u_{0}$ has no zeros and that $u_{1}$ has exactly one zero on any half-open interval of length 1 ). By changing signs as necessary we can also assume that $u_{0}>0$ and that $u_{1}(x)>0$ on $(0,1)$. Since $u_{0}(0)=u_{0}(1)>0$, $u_{1}(0)=0=u_{1}(1)$, and $u_{0}$ and $u_{1}$ are normalized on $[0,1]$, it is clear that $u_{1}(x)^{2}-u_{0}(x)^{2}$ must change signs on $(0,1)$ and, furthermore, that there are points $a, b \in(0,1)$ with $a<b$ such that $u_{1}(x)=u_{0}(x)$ at $x=a, b$ and $u_{1}(x)<u_{0}(x)$ on $[0, a)$ and on $(b, 1]$. We now argue that $u_{1}(x)>u_{0}(x)$ for $x \in(a, b)$. There are two cases to consider: (i) $u_{1}(x)<u_{0}(x)$ on $(c, d) \subset$ $(a, b)$ and $u_{1}(c)=u_{0}(c), u_{1}(d)=u_{0}(d)$, (ii) $u_{1}(c)=u_{0}(c), u_{1}^{\prime}(c)=u_{0}^{\prime}(c)$ for some $c \in(a, b)$ but $u_{1}(x)>u_{0}(x)$ on a neighborhood of $c$. Case (i) is eliminated by using $u_{0}-u_{1}$ as a trial function in the Rayleigh-Ritz inequality as follows:

$$
\begin{aligned}
\lambda_{1}^{\prime}=\mu_{1}\left(q_{*} ;[0,1]\right) & <\mu_{1}\left(q_{*} ;[c, d]\right) \\
& \leq \frac{\int_{c}^{d}\left(u_{0}-u_{1}\right)\left[\left(-\frac{d^{2}}{d x^{2}}+q_{*}(x)\right)\left(u_{0}-u_{1}\right)\right] d x}{\int_{c}^{d}\left(u_{0}-u_{1}\right)^{2} d x}
\end{aligned}
$$


so

$$
\begin{aligned}
\lambda_{1}^{\prime} \int_{c}^{d}\left(u_{0}-u_{1}\right)^{2} d x & <\int_{c}^{d}\left(u_{0}-u_{1}\right)\left(\lambda_{0} u_{0}-\lambda_{1}^{\prime} u_{1}\right) d x \\
& =\lambda_{0} \int_{c}^{d}\left(u_{0}-u_{1}\right)^{2} d x-\left(\lambda_{1}^{\prime}-\lambda_{0}\right) \int_{c}^{d}\left(u_{0}-u_{1}\right) u_{1} d x
\end{aligned}
$$

which implies $\lambda_{1}^{\prime}<\lambda_{0}$ and contradicts equation (2). (Here $\mu_{1}\left(q_{*} ;[c, d]\right)$ denotes the first Dirichlet eigenvalue for $-d^{2} / d x^{2}+q_{*}(x)$ acting on $L^{2}(c, d)$ with Dirichlet boundary conditions at $c$ and $d$.) To eliminate case (ii) we observe that in that case

$$
\left(u_{1}-u_{0}\right)(c)=0, \quad\left(u_{1}-u_{0}\right)^{\prime}(c)=0,
$$

and

$$
\left(u_{1}-u_{0}\right)^{\prime \prime}(c)=\left.\left[q\left(u_{1}-u_{0}\right)+\lambda_{0} u_{0}-\lambda_{1}^{\prime} u_{1}\right]\right|_{x=c}=-\left(\lambda_{1}^{\prime}-\lambda_{0}\right) u_{0}(c)<0,
$$

and that these are incompatible with $u_{1}(x)>u_{0}(x)$ in a neighborhood of $c$. This establishes that $B_{-} \cap[0,1]=(a, b)$ and hence the theorem.

From this theorem we can easily obtain corresponding results about $\nu_{1}(q)-$ $\nu_{0}(q)$ and $\mu_{1}(q)-\nu_{0}(q)$ for the operator $-d^{2} / d x^{2}+q(x)$ on $L^{2}(0,1)$ where $q$ is allowed to vary in the class $S(M)=\left\{q \in L^{\infty}(0,1)\|\| q \|_{\infty} \leq M\right\}, M>0$. One obtains

Theorem 2. With notation as given above there exist minimizing potentials $q_{*}$ for $\mu_{1}(q)-\nu_{0}(q)$ and $\nu_{1}(q)-\nu_{0}(q)$, and any such minimizer is of the form

$$
q_{*}(x)= \pm M\left[\chi_{J}(x)-\chi_{[0,1] \backslash J}(x)\right] \text { a.e. },
$$

where $J$ is a symmetric interval $(a, 1-a), 0<a<\frac{1}{2}$, and where the plus sign applies for minimizers of $\nu_{1}-\nu_{0}$ and the minus sign applies for minimizers of $\mu_{1}-\nu_{0}$. Moreover, for a given $M$ the minimal eigenvalue gaps are all equal,

$$
\min _{S(M)}\left(\mu_{1}-\nu_{0}\right)=\min _{S(M)}\left(\nu_{1}-\nu_{0}\right)=\underset{\widetilde{S}(M)}{\min }\left(\lambda_{1}^{\prime}-\lambda_{0}\right) .
$$

Proof. Existence of minimizing potentials $q_{*}$ was outlined above. The remaining arguments are identical for $\nu_{1}-\nu_{0}$ and $\mu_{1}-\nu_{0}$ so for concreteness we shall concentrate on $\nu_{1}-\nu_{0}$.

Assume $q_{*}$ minimizes $\nu_{1}-\nu_{0}$ over $q \in S(M)$. We extend $q_{*}$ to a potential $\tilde{q}_{*}$ on $\mathbb{R}$ by periodicity and then consider the Hill's equation problem for $\tilde{q}_{*}$. The inequalities given in (3) now show that

$$
\nu_{1}\left(q_{*}\right)-\nu_{0}\left(q_{*}\right)=\nu_{1}\left(\tilde{q}_{*}\right)-\nu_{0}\left(\tilde{q}_{*}\right) \geq \lambda_{1}^{\prime}\left(\tilde{q}_{*}\right)-\lambda_{0}\left(\tilde{q}_{*}\right)
$$

so that

$$
\min _{q \in S(M)}\left[\nu_{1}(q)-\nu_{0}(q)\right] \geq \min _{q \in \widetilde{S}(M)}\left[\lambda_{1}^{\prime}(q)-\lambda_{0}(q)\right]
$$

On the other hand we can also demonstrate the reverse inequality as follows. Letting $Q_{*}$ be a minimizer for $\lambda_{1}^{\prime}-\lambda_{0}$ on $\widetilde{S}(M)$ we observe that there are two distinct translates of $Q_{*}$ that are symmetric about $x=0$ (and also about $\left.x=\frac{1}{2}\right)$. This follows from our characterization of minimizers of $\lambda_{1}^{\prime}-\lambda_{0}$ as given in Theorem 1. From the theory of Hill's equation with a symmetric 
potential [9] it follows that $\lambda_{1}^{\prime}$ coincides with $\nu_{1}$ for one of these symmetric translates and with $\mu_{1}$ for the other and that $\lambda_{0}=\nu_{0}$ for both of them. This shows that one of these symmetric translates $Q_{1}$ actually yields equality in

$$
\nu_{1}\left(Q_{1}\right)-\nu_{0}\left(Q_{1}\right)=\underset{\widetilde{S}(M)}{\min }\left(\lambda_{1}^{\prime}-\lambda_{0}\right)
$$

and hence that

$$
\min _{S(M)}\left(\nu_{1}-\nu_{0}\right)=\underset{\widetilde{S}(M)}{\min }\left(\lambda_{1}^{\prime}-\lambda_{0}\right) .
$$

Similarly, the other symmetric translate $Q_{2}$ of $Q_{*}$ yields

$$
\mu_{1}\left(Q_{2}\right)-\nu_{0}\left(Q_{2}\right)=\underset{\widetilde{S}(M)}{\min }\left(\lambda_{1}^{\prime}-\lambda_{0}\right)
$$

and hence that

$$
\min _{S(M)}\left(\mu_{1}-\nu_{0}\right)=\underset{\widetilde{S}(M)}{\min }\left(\lambda_{1}^{\prime}-\lambda_{0}\right)
$$

This establishes (18).

Since any minimizer of $\nu_{1}-\nu_{0}\left(\right.$ or $\left.\mu_{1}-\nu_{0}\right)$ gives a minimizer of $\lambda_{1}^{\prime}-\lambda_{0}$ via periodic extension of period 1 , it is clear that if $q_{*}$ minimizes $\nu_{1}-\nu_{0}$ (or $\mu_{1}-\nu_{0}$ ) then its periodic extension $\tilde{q}_{*}$ must meet the conditions given in Theorem 1. To see that it also must be symmetric with respect to $x=\frac{1}{2}$ we argue by contradiction. For this purpose, suppose that $q_{*}$ is not symmetric with respect to $x=\frac{1}{2}$ and consider the eigenfunction $\varphi_{1}$ corresponding to the eigenvalue $\mu_{1}$ (respectively $\nu_{1}$ ). For the periodically extended problem it is clear that $\varphi_{1}$ is a Floquet solution and that its Floquet multiplier $\rho$ is real and negative as is given by

$$
\left.\rho=\varphi_{1}^{\prime}(1) / \varphi_{1}^{\prime}(0) \quad \text { (resp., } \varphi_{1}(1) / \varphi_{1}(0)\right) .
$$

Now either $\rho \neq-1$, in which case $\mu_{1}$ (resp., $\nu_{1}$ ) cannot be in the spectrum of the associated Hill operator, or $\rho=-1$, and $\varphi_{1}$ is an antiperiodic characteristic function of the Hill operator. In the first case it fo!lows that $\mu_{1}>\lambda_{1}^{\prime}$ (resp., $\nu_{1}>$ $\lambda_{1}^{\prime}$ ) and hence $\mu_{1}-\nu_{0}>\lambda_{1}^{\prime}-\lambda_{0}$ (resp., $\nu_{1}-\nu_{0}>\lambda_{1}^{\prime}-\lambda_{0}$ ), contradicting equation (18). In the second case, a contradiction still arises since in that case $\varphi_{1}$ would have to be a second independent antiperiodic characteristic function for Hill's equation with $\mu_{1}=\lambda_{1}^{\prime}$ (resp., $\nu_{1}=\lambda_{1}^{\prime}$ ), and this would imply that $\lambda_{2}^{\prime}=\lambda_{1}^{\prime}$, i.e., that $\lambda_{1}^{\prime}$ is degenerate with $\lambda_{2}^{\prime}$, which we know from the proof of Theorem 1 to be impossible. The independence of $\varphi_{1}$ and $u_{1}$ (the characteristic function for $\lambda_{1}^{\prime}$ ) follows from the fact that the zeros of $u_{1}$ (and of $u_{1}^{\prime}$ ) may be assumed via the theory of Hill's equation with a symmetric potential [9] to have the same symmetry as the potential, whereas the zeros of $\varphi_{1}$ do not share this symmetry because of our original assumption. This contradiction shows that the minimizers of $\mu_{1}-\nu_{0}$ (or $\left.\nu_{1}-\nu_{0}\right)$ must be symmetric about $x=\frac{1}{2}$, thereby completing the proof.

\section{APPENDIX}

In this appendix we sketch the argument showing that $\lambda_{1}^{\prime}\left(q_{*}\right)$ must be a nondegenerate eigenvalue. A similar argument appears in [4]. The argument 
is similar to the argument for the nondegenerate case given in our proof of Theorem 1 above, except that it requires a more elaborate set-up and somewhat different conclusions must be reached (as we desire to show that the degenerate case cannot occur).

Let $\left\{\psi_{i}\right\}_{i=1}^{r}$ be an orthonormal eigenbasis for $\lambda_{1}^{\prime}$ where we assume $r>1$ (for the one-dimensional problem dealt with in this paper this implies that $r$ is necessarily 2, but we shall give the argument for arbitrary $r$ since it is no more difficult in this generality). For any perturbation $P(x)$ the potential $q_{*}+\kappa P$ gives rise to $r$ eigenvalues (not necessarily distinct) $\left\{\lambda_{1, i}^{\prime}(\kappa)\right\}_{i=1}^{r}$, each an analytic function of $\kappa$ satisfying $\lambda_{1, i}^{\prime}(0)=\lambda_{1}^{\prime}\left(q_{*}\right)$. Moreover, the derivatives

$$
\left.\frac{d}{d \kappa}\left[\lambda_{1, i}^{\prime}(\kappa)-\lambda_{0}\left(q_{*}+\kappa P\right)\right]\right|_{\kappa=0}
$$

are given by the eigenvalues of the $r \times r$ selfadjoint matrix $A$ with entries

$$
\begin{aligned}
a_{i j} & =\int_{0}^{1} P(x)\left[\overline{\psi_{i}(x)} \psi_{j}(x)-\left|u_{0}(x)\right|^{2} \delta_{i j}\right] d x \\
& =\left(\psi_{i}, P \psi_{j}\right)-\left(u_{0}, P u_{0}\right) \delta_{i j} .
\end{aligned}
$$

For a perturbation $P$ such that $q_{*}+\kappa P \in \widetilde{S}(M)$ for some interval $[0, \varepsilon)$, $\varepsilon>0$ (henceforth expressed as " $P$ is admissible for positive $\kappa$ " or " $P$ is right-admissible"), it follows that $A$ must be positive semidefinite; otherwise we could decrease $\lambda_{1}^{\prime}-\lambda_{0}$ from its value at $q_{*}$ since

$$
\begin{aligned}
\frac{d}{d \kappa} & {\left.\left[\lambda_{1}^{\prime}\left(q_{*}+\kappa P\right)-\lambda_{0}\left(q_{*}+\kappa P\right)\right]\right|_{\kappa=0} } \\
& =\left.\min _{1 \leq i \leq r} \frac{d}{d \kappa}\left[\lambda_{1, i}^{\prime}(\kappa)-\lambda_{0}\left(q_{*}+\kappa P\right)\right]\right|_{\kappa=0},
\end{aligned}
$$

where the expression on the left denotes a right-hand derivative. Thus if $u$ denotes any normalized eigenfunction for $\lambda_{1}^{\prime}\left(q_{*}\right)$ we have

$$
u=\sum_{i=1}^{r} c_{i} \psi_{i}, \quad \text { where } \sum_{i=1}^{r}\left|c_{i}\right|^{2}=1
$$

and hence

$$
\begin{aligned}
\int_{0}^{1} P(x)\left[|u(x)|^{2}-\left|u_{0}(x)\right|^{2}\right] d x \\
\quad=\sum_{1 \leq i, j \leq r} \overline{c_{i} c_{j}} \int_{0}^{1} P(x)\left[\overline{\psi_{i}(x)} \psi_{j}(x)-\left|u_{0}(x)\right|^{2} \delta_{i j}\right] d x=(c, A c) \geq 0 .
\end{aligned}
$$

It follows that if $P$ is admissible for positive $\kappa$, then

$$
\int_{0}^{1} P(x)\left[|u(x)|^{2}-\left|u_{0}(x)\right|^{2}\right] d x \geq 0
$$

for any normalized eigenfunction $u$ of $\lambda_{1}^{\prime}$. Similarly, one can show that if $P$ is admissible for negative $\kappa$ (or left-admissible), then

$$
\int_{0}^{1} P(x)\left[|u(x)|^{2}-\left|u_{0}(x)\right|^{2}\right] d x \leq 0
$$

for any normalized eigenfunction $u$ of $\lambda_{1}^{\prime}$. 
Now define for any normalized eigenfunction $u$ of $\lambda_{1}^{\prime}$, the sets $B_{+}(u) \equiv$ $\left\{\left.x \in \mathbb{R}|| u_{0}(x)\right|^{2}>|u(x)|^{2}\right\}$ and $B_{-}(u) \equiv\left\{\left.x \in \mathbb{R}|| u_{0}(x)\right|^{2}<|u(x)|^{2}\right\}$. It follows from the inequalities above that a positive perturbation $P$ supported on $B_{+}(u)$ cannot be admissible for positive $\kappa$ and neither can a positive perturbation $P$ supported on $B_{-}(u)$ be admissible for negative $\kappa$. Neglecting some measuretheoretic technicalities (see [4] for details) it follows that $q_{*}=M$ on $B_{+}(u)$ and $q_{*}=-M$ on $B_{-}(u)$ for any $u$. Thus,

$$
q_{*}(x)=M\left[\chi_{B_{+}(u)}(x)-\chi_{B_{-}(u)}(x)\right]
$$

for any $u$. However, this can hardly be the case for two linearly independent normalized eigenfunctions $u$. In particular, suppose that $\eta_{1}$ and $\eta_{2}$ denote two such $u$ 's that are orthogonal to each other. Since neither $B_{+}(u)$ nor $B_{-}(u)$ can be empty (by the fact that $u_{0}$ and $u$ are always taken to be normalized on $(0,1)$ and are continuous) it is clear that we can find a point $x_{0}$ at which $\eta_{1}^{2}\left(x_{0}\right)=\eta_{2}^{2}\left(x_{0}\right)=u_{0}^{2}\left(x_{0}\right) \neq 0$. By adjusting signs as necessary we may assume that $\eta_{1}\left(x_{0}\right)=\eta_{2}\left(x_{0}\right)=u_{0}\left(x_{0}\right)>0$. But now consider a third normalized eigenfunction for $\lambda_{1}^{\prime}$,

$$
\eta_{3}=\left(\eta_{1}-\eta_{2}\right) / \sqrt{2} \text {. }
$$

Clearly, $\eta_{3}\left(x_{0}\right)=0<u_{0}\left(x_{0}\right)$, and therefore, using the continuity of all the eigenfunctions involved, it follows that $B_{ \pm}\left(\eta_{3}\right)$ cannot coincide with $B_{ \pm}\left(\eta_{1}\right)$ and $B_{ \pm}\left(\eta_{2}\right)$, and hence the relation (33) above cannot hold for $u=\eta_{3}$. This contradiction shows that $\lambda_{1}^{\prime}$ cannot have two linearly independent eigenfunctions, i.e., that $\lambda_{1}^{\prime}$ cannot be degenerate. Hence, $r=1$ and $\lambda_{1}^{\prime}$ is nondegenerate (simple), thus completing the proof.

\section{ACKNOWLEDGMENTS}

The first author is grateful to the Mathematics Department at the University of Tennessee for its hospitality during the time that this work was in progress. We would also like to thank the referee for helpful comments and suggestions.

\section{REFERENCES}

1. M. S. Ashbaugh, Optimization of the characteristic values of Hill's equation subject to a p-norm constraint on the potential, J. Math. Anal. Appl. 143 (1989), 438-447.

2. M. S. Ashbaugh and E. M. Harrell, Potentials having extremal eigenvalues subject to $p$ norm constraints (Proc. 1984 Workshop on Spectral Theory of Sturm-Liouville Differential Operators) (H. G. Kaper and A. Zettl, eds.), ANL-84-73, Argonne National Laboratory, Argonne, IL, 1984, pp. 19-29. (Available from National Technical Information Service, Springfield, VA.)

3. __ Maximal and minimal eigenvalues and their associated nonlinear equations, J. Math. Phys. 28 (1987), 1770-1786.

4. M. S. Ashbaugh, E. M. Harrell, and R. Svirsky, On minimal and maximal eigenvalue gaps and their causes, Pacific J. Math. 147 (1991), 1-24.

5. J. A. Dieudonné, Infinitesimal calculus, Hermann, Paris, 1971.

6. H. Egnell, Extremal properties of the first eigenvalue of a class of elliptic eigenvalue problems, Ann. Scuola Norm. Sup. Pisa Cl. Sci. (4) 14 (1987), 1-48.

7. E. L. Ince, Ordinary differential equations, Dover, New York, 1956.

8. T. Kato, Perturbation theory for linear operators, 2nd ed., Springer-Verlag, Berlin, 1976. 
9. W. Magnus and S. Winkler, Hill's equation, Dover, New York, 1979.

10. M. Reed and B. Simon, Methods of modern mathematical physics, Vol. IV: Analysis of operators, Academic Press, New York, 1978.

Department of Mathematics, University of Missouri, Columbia, Missouri 65211

E-mail address: MATHMSA@umcvmb.bitnet

Department of Mathematics, University of Tennessee, Knoxville, Tennessee 379961300

E-mail address: RSVIRSKY@utkvm1.bitnet 